\title{
E-solid regular semigroups and solid binary algebras
}

\author{
RAYMOND E. BROEKSTEEG
}

The idempotents of a regular semigroups play an important role in determining the semigroup's structure, and so have been studied by many authors. In $[7,8]$ Nambooripad defined a regular biordered set to be a nonempty set together with a partial binary operation defined on it that satisfied certain conditions. He proceeded to show that regular biordered sets characterise the idempotents of regular semigroups.

For the special case of a locally inverse regular semigroup, Nambooripad [9] found that one may abstractly characterise its set of idempotents as a binary algebra which satisfies a given finite list of identities (where a binary algebra is a nonempty set together with a binary operation defined on it). We call this type of binary algebra a local semilattice algebra; other names used elsewhere in the literature include 'partially associative pseudosemilattice', 'local semilattice', and 'pseudosemilattice'.

The main purpose of this thesis is to derive a similar characterisation of the idempotents of E-solid regular semigroups (which will coincide with the usual band of idempotents if the semigroup is orthodox). This characterisation will be called a solid binary algebra. We also establish a concept of variety for regular biordered sets which is analogous to the concept of existence variety (e-variety) introduced by Hall [5].

Chapter 1 contains preliminary results on semigroups, biordered sets, and evarieties.

In Chapter 2, we give the promised characterisation of the set of idempotents of an E-solid regular semigroup. We first define a solid binary algebra to be a binary algebra which satisfies six given identities, and their duals. For an E-solid regular semigroup $S$, we define a binary operation $*$ on $E(S)$ by

$$
(\forall e, f \in E(S)) e * f=\text { the identity element of the group } H_{e f}^{S} \text {. }
$$

It is shown that $(E(S), *)$ is a solid binary algebra, which we call the solid binary algebra of $S$. Conversely, we show that each solid binary algebra is the solid binary algebra of some E-solid regular semigroup.

Received 15th August, 1994

Thesis submitted to Monash University, February 1994. Degree approved July 1994. Supervisor: Dr T.E. Hall.

Copyright Clearance Centre, Inc. Serial-fee code: 0004-9729/95 \$A2.00+0.00. 
We show that morphisms between solid binary algebras are precisely the morphisms between E-solid regular semigroups with their domains restricted. For the solid binary algebra $(E(S), *)$ of an E-solid regular semigroup $S$, we prove that the subalgebras of $(E(S), *)$ are in a one-to-one correspondence with the idempotent-generated regular subsemigroups of $S$. We also prove that the solid binary algebra of the direct product of a family of E-solid regular semigroups is the direct product of the solid binary algebras of the members of the family.

In Chapter 3, we consider the structure of solid binary algebras. We show that each solid binary algebra is a semilattice of rectangular bands, and we use this result to establish a structure theorem for solid binary algebras which is remarkably similar to the structure theorem for bands due to Petrich [6, Theorem IV.3.16]. We also show that the free solid binary algebra can be embedded in a free completely regular semigroup; thus the word problem for the free solid binary algebra can be solved using a solution of the word problem for the free completely regular semigroup.

In Chapter 4, a bivariety of regular biordered sets is defined to be a nonempty class of regular biordered sets which is closed under taking direct products, regular bimorphic images and relatively regular biordered subsets. Examples of bivarieties include the class of all solid regular biordered sets and the class of all regular local semilattices. We prove that the class of all the biordered sets of the members of an e-variety of regular semigroups is a bivariety of regular biordered sets. We then show that sending e-varieties to bivarieties in this way defines a complete lattice morphsim which maps the complete lattice of all e-varieties of regular semigroups onto the complete lattice of all bivarieties of regular biordered sets. By restricting this mapping to the E-solid e-varieties, we obtain a complete lattice morphism which maps the complete lattice of all e-varieties of E-solid regular semigroups onto the complete lattice of all varieties of solid binary algebras. Similarly, by restricting the mapping to locally inverse e-varieties, we obtain a complete lattice morphism which maps the complete lattice of all e-varieties of locally inverse regular semigroups onto the complete lattice of all varieties of local semilattice algebras. (This latter result had already been proved, using a different approach, by Auinger [1].) Finally, for each set $X$ with at least two elements, we show that a bivariety contains a free object on $X$ if and only if it consists entirely of solid regular biordered sets or entirely of regular local semilattices.

The contents of Chapters 2,3 and 4 have individually been rewritten as papers for publication $[2,3,4]$.

\section{References}

[1] K. Auinger, 'On the lattice of existence varieties of locally inverse semigroups', Canad. Math. Bull. 37 (1994), 13-20. 
[2] R. Broeksteeg, 'The set of idempotents of a completely regular semigroup as a binary algebra', Bull. Austral. Math. Soc. 50 (1994), 91-107.

[3] R. Broeksteeg, 'The structure of solid binary algebras', (preprint).

[4] R. Broeksteeg, 'A concept of variety for regular biordered sets', Semigroup Forum (to appear).

[5] T.E. Hall, 'Identities for existence varieties of regular semigroups', Bull. Austral. Math. Soc. 40 (1989), 59-77.

[6] J.M. Howie, An introduction to semigroup theory, London Math. Soc. Monographs 7 (Academic Press, London, New York, 1976).

[7] K.S.S. Nambooripad, 'Structure of regular semigroups I: Fundamental regular semigroups', Semigroup Forum 9 (1975), 354-363.

[8] K.S.S. Nambooripad, 'Structure of regular semigroups I', Mem. Amer. Math. Soc. 22 (1979).

[9] K.S.S. Nambooripad, 'Pseudo-semilattices and biordered sets I', Simon Stevin 55 (1981), 103-110.

\section{Mathematics Department}

Monash University

Clayton Vic 3168

Australia 